“C 2017 IEEE. Personal use of this material is permitted. Permission from IEEE must be obtained for all other uses, in any current or future media, including reprinting/republishing this material for advertising or promotional purposes, creating new collective works, for resale or redistribution to servers or lists, or reuse of any copyrighted component of this work in other works." 


\title{
Joint Communications and Sensing Using Two Steerable Analog Antenna Arrays
}

\author{
J. Andrew Zhang ${ }^{1}$, Antonio Cantoni ${ }^{2}$, Xiaojing Huang ${ }^{1}$, Y. Jay Guo ${ }^{1}$ and Robert W. Heath $\mathrm{Jr}^{3}$ \\ ${ }^{1}$ University of Technology Sydney, Global Big Data Technologies Centre (GBDTC), Australia \\ ${ }^{2}$ University of Western Australia, Perth, Australia \\ ${ }^{3}$ The University of Texas at Austin, Austin, TX 78712, USA \\ \{Andrew.Zhang; Xiaojing.Huang; Jay.Guo\}@uts.edu.au; antonio.cantoni@uwa.edu.au; rheath@utexas.edu.
}

\begin{abstract}
Beam-steering has great potentials for joint communications and sensing, which is becoming a demanding feature on many emerging platforms such as unmanned aerial vehicles and smart cars. Although beam-steering has been extensively studied for communications and radar sensing respectively, its application in the joint system is not straightforward due to different beamforming requirements by communications and sensing. In this paper, we propose a low-cost system framework which allows seamless operation of communications and sensing, using two small-size steerable analog antenna arrays. We provide system architecture, high-level protocols, detailed signal model, novel beamforming design and advanced 1D compressive sensing algorithms for joint communications and sensing. We also provide preliminary simulation results which validate the effectiveness of the proposed technique in resolving closely located objects.
\end{abstract}

\section{INTRODUCTION}

There have been increasing demands for systems with both communications and (radar) sensing capabilities, on emerging platforms such as unmanned aerial vehicles and smart cars [1], [2]. Sensing techniques are evolving towards advanced pattern analysis, based on estimating the position and speed of objects in surrounding environment. Instead of having two separate systems, it is possible to develop joint communication and sensing (JCAS) techniques to integrate the two functions into one system by sharing hardware and signal processing modules, and achieve immediate benefits of reduced cost, size, weight, and better spectrum efficiency. An integrated system will also benefit from mutual sharing of information for improved performance, e.g., using sensed environment knowledge to assist beamforming (BF) design [3].

Although speed and distance estimation has been well studied for JCAS [4], direction estimation remains as a challenging problem for portable devices. Beam-steering could be a viable solution to this problem. It can also improve the sensing capability for remote objects whose signals would otherwise be buried in those from nearby objects. Beamsteering for communications and radar sensing respectively has been studied extensively. However, its applications in JCAS, particularly for portable devices, is not straightforward. The main challenge is that communications and sensing have different requirements for BF. Devices having small antenna

A. Cantoni's work is supported by Discovery Project DP140100522 of the Australian Research Council. arrays generally work on high frequency bands and hence have large propagation loss. In this case, sensing requires timevarying directional scanning beams, while communications require stable and accurately-pointed beams to achieve large BF gain.

In this paper, we present a framework for JCAS using beam-steering that allows seamless operation of JCAS, and propose novel $\mathrm{BF}$ design to enable accurate sensing without compromising communication performance. To our best knowledge, this is the first work that investigates how to integrate analog BF into a JCAS system with multicarrier modulation and packet transmission. Our main contributions are as follows: (1) we propose to use two steerable analog antenna arrays to balance system performance and complexity. These analog arrays use digitally controlled phase shifters for beam steering, as well as attenuators when necessary which enable more flexible beam generation. Using two arrays can not only reduce leakage signal from the transmitter to receiver, which is essential for making sensing work efficiently, but also provide great flexibility in designing $\mathrm{BF}$ and operation modes; (2) Instead of generating a conventional single beam, we propose a system architecture and protocol which exploits arrays' multibeam capacity, and investigate how to configure beams to meet and balance the different requirements for sensing and communications; and (3) we formulate the signal processing and parameter estimation problems mathematically, and propose advanced 1D Bayesian Compressive Sensing (BCS)-based solutions which are very efficient in resolving closely located objects using overcomplete dictionaries. We also present preliminary simulation results that validate the effectiveness of the proposed framework.

\section{PRoposed FrameWORK}

We consider a system where two nodes perform twoway point-to-point communications in time division duplex (TDD) mode, and simultaneously sensing the environment to determine locations and speed of nearby objects. Using TDD enables better hardware sharing and avoids complex synchronization between two-way transmissions, compared to frequency division duplex. Each node uses two steerable antenna arrays. One array is dedicated to the receiver, the other is shared by transmitter and receiver through time division. We 


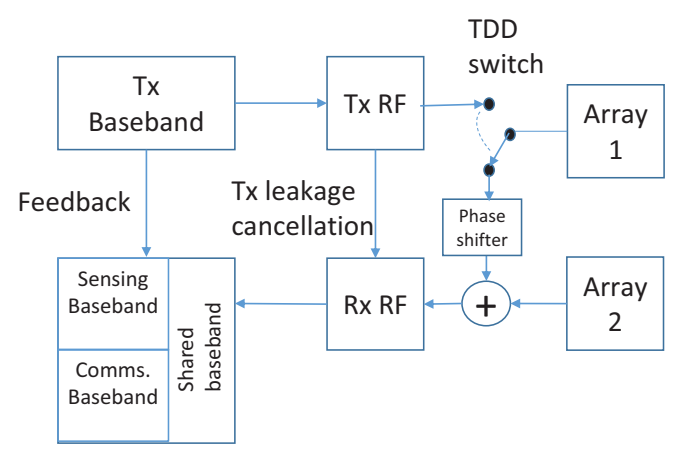

Fig. 1. Block diagram of the proposed transceiver.

consider orthogonal frequency division multiplexing (OFDM) here for its popularity in modern communications, and its strong potential for sensing [4]. The proposed framework can be extended to other packet-based communication systems.

\section{A. System Architecture}

Fig. 1 shows the diagram of the proposed transceiver. The transmitter baseband module is common to communications and sensing. The baseband signal is sent to the transmitter radio frontend (RF). An electronic switch chooses to connect Array 1 to transmitter or receiver. The transmitter RF signal after power amplifier can also be optionally fed to the receiver $\mathrm{RF}$ for cancelling leakage signal from the transmitter.

At the receiver, Array 2 is always connected to the RF, and Array 1 is only connected through a digitally controlled phase shifter when the node itself is not transmitting and only receiving signal from the other node. Adding Array 1 can ideally double the $\mathrm{BF}$ gain in this case, where signals from the two arrays are constructively added together by a combiner before the RF module. Sensing and communications at the receiver baseband share some processings such as discrete Fourier transform (DFT), channel estimation and equalization, but are largely different. The receiver baseband also accepts feedback from the transmitter baseband, mainly for getting a clear sensing signal by removing the data symbols from the received signal.

Fig. 2 illustrates the proposed procedure of JCAS between two nodes A and B. From the receiver's viewpoint, one complete cycle for each node includes two stages: active sensing, and communications and passive Sensing. We refer active and passive sensing to the cases where sensing signal is transmitted by the node itself and by other nodes, respectively. The major difference between them is that the transmitted signal is known to the receiver in the former while it is typically unknown, but may be decoded, in the latter. For simplicity, we will call them as sensing and communications stages hereafter. Using Node A as an example, we describe the detailed implementations in the two stages below.

In the sensing stage of Node A, when Node B is in the communications stage, the transmitter uses Array 1 to form multiple beams, with one beam pointing to Node B and other beams adapting to the sensing requirement. Array 2 of Node

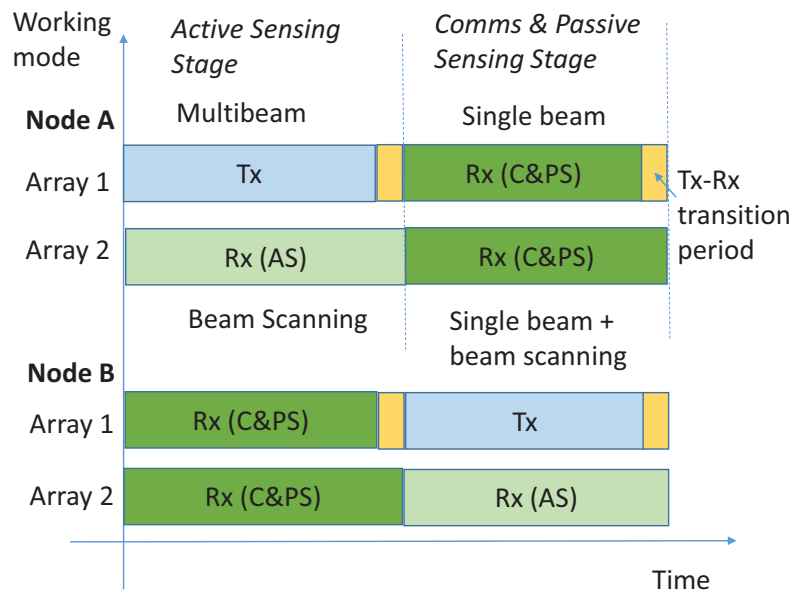

Fig. 2. Procedure of communications and sensing in a point-to-point scenario.

A is used for sensing only. It typically forms a narrow singlebeam and scans the environment. At the end of sensing stage, there is a short transition period between transmission and reception, as usually exists in a TDD transceiver. This period also serves as a guard interval for Array 2, such that the reflected signals from its own transmitter will be separated from the received signals from Node B's transmitter in the coming communications period.

In the communications stage when Node B is in the sensing stage, both Arrays 1 and 2 of Node A work in the receiving mode, and their signals are combined and processed, primarily for communications, and optionally for sensing. Sensing in this case uses transmitted signal from Node B.

\section{B. Formulation of Signal Model}

We consider uniform linear antenna arrays and planar wavefront in this paper. Each array has $M$ antennas equally spaced at $d$. The array response vector for an angle-of-departing (AoD) $\theta_{t}$ or angle-of-arrival (AoA) $\theta_{r}$ is given by

$$
\mathbf{a}(\theta)=\left[1, e^{-j \kappa \sin (\theta)}, \cdots, e^{-j \kappa(M-1) \sin (\theta)}\right]^{H},
$$

where $\kappa=2 \pi d / \lambda$ and $\lambda$ is the wavelength, and $\theta$ is for either $\theta_{t}$ or $\theta_{r}$.

In the OFDM system, let $N$ denote the number of subcarriers and $B$ the total bandwidth. Then we get the subcarrier interval $f_{0}=B / N$ and OFDM symbol period $T_{s}=N / B+T_{p}$ where $T_{p}$ is the period of cyclic prefix. The baseband signal in the transmitter can then be represented as

$$
\mathbf{s}=\mathbf{F}^{H} \tilde{\mathbf{s}},
$$

where $\tilde{\mathbf{s}}$ is the $N \times 1$ data symbol vector, $\mathbf{F}$ is the DFT matrix, and $\mathbf{s}$ is the time domain signal. Let $s(t)$ denote the time-domain baseband signal with cyclic prefix appended. The signal transmitted from the antenna array is

$$
\mathbf{x}(t)=s(t) \mathbf{w}_{t},
$$

where $\mathbf{w}_{t}$ is the transmitter $\mathrm{BF}$ vector.

For sensing, consider $L$ reflected signals by obstacles, with AoDs and AoAs $\theta_{t, \ell}$ and $\theta_{r, \ell}$, respectively. The time-varying 
physical channels between the $M$ transmitting and receiving antennas can then be represented as

$$
\mathbf{H}=\sum_{\ell=1}^{L} b_{\ell} \delta\left(t-\tau_{\ell}\right) e^{j 2 \pi f_{D, \ell} t} \mathbf{a}^{T}\left(\theta_{t, \ell}\right) \otimes \mathbf{a}\left(\theta_{r, \ell}\right),
$$

where for the $\ell$-th multipath, $b_{\ell}$ is its amplitude, $\tau_{\ell}$ is the propagation delay, and $f_{D, \ell}$ is the associated Doppler frequency that causes time varying of $\mathbf{H}$.

For communications, we can use the same expression as (4) with different parameter values. These multipath signals are caused more likely due to different propagation mechanisms such as diffraction and scattering.

Let the receiver $\mathrm{BF}$ vector be $\mathbf{w}_{r}$. The signal arriving at a receiver array for either sensing or communications can be represented as

$$
\begin{aligned}
& y(t) \\
& =\sum_{\ell=1}^{L} b_{\ell} e^{j 2 \pi f_{D, \ell} t}\left(\mathbf{w}_{r}^{T} \mathbf{a}\left(\theta_{r, \ell}\right)\right) \cdot\left(\mathbf{a}^{T}\left(\theta_{t, \ell}\right) \mathbf{w}_{t}\right) s\left(t-\tau_{\ell}\right)+z(t)
\end{aligned}
$$

where $z(t)$ is AWGN.

The processing for communications are similar to that in conventional OFDM systems. For sensing, we will look into more details in Section IV.

\section{DESIGN OF BF VECTORS}

Now we want to design BF vectors to meet both the communication and sensing requirements, that is, stable and high-gain beams for communications without demanding complex channel tracking, and scanning beams to enable accurate estimation of time delay, Doppler frequency and AoAs/AoDs for sensing. We assume a packet-based communication system where each packet contains many OFDM symbols, and each node sends multiple packets to the other node in one cycle.

\section{A. Basic Requirements}

Firstly, there are two basic requirements for BF vectors $\mathbf{w}_{t}$ and $\mathbf{w}_{r}$ :

- $\mathbf{w}_{t}$ needs to be fixed during at least one packet to make the channel stable for communications (This can be relaxed if powerful channel tracking is available);

- For sensing $\mathbf{w}_{r}$ needs to be fixed during at least one OFDM symbol so that information symbols can be removed from the received signal; for communications, it needs to be fixed over the whole packet.

These two requirements can be generally satisfied, e.g., in a typical mmWave system. Consider an OFDM system with carrier frequency $24 \mathrm{GHz}$, bandwidth $B=80 \mathrm{MHz}$ and $N=128$ subcarriers. The OFDM symbol period is about $T_{s}=1.6 u$ s. Within $1 \mathrm{~ms}$ which approximately equals to the length of 600 OFDM symbols, the moving distance is less than $5 \mathrm{~cm}$ for a relative speed smaller than $50 \mathrm{~m} / \mathrm{s}$. With $f_{D, \ell} T_{s}<0.0064$, the Doppler phase shift can be regarded as unchanged over multiple $T_{s} \mathrm{~s}$. For more details on the impact of OFDM parameters on sensing, the readers are referred to [4].

Secondly, to ensure sufficient power for communications, at least one directional beam pointing to the target node is required, similarly for the receiver in the communications stage. We want transmitter scanning beam to be directional too, so that sufficient energy could be reflected for sensing. Their directivity can also assist the coarse estimation of AoDs.

\section{B. Our BF Design}

Assume that each packet has $N_{r} N_{d}$ consecutive OFDM symbols, indexed from $1, \cdots, N_{r} N_{d}$. Considering the basic requirements above, our proposed BF design are as follows.

(1) Fix $\mathbf{w}_{t}$ during one packet. Design $\mathbf{w}_{t}$ to generate two directional beams. One always points to the target node for communications, and the other points to a direction for sensing. The sensing beam scans the whole directions of interest over $N_{t}$ packet periods. The width and gain of the scanning beam can be different from the communication beam which also implicitly senses a fixed direction;

(2) For sensing, design $N_{r}$ different $\mathbf{w}_{r} s$, and apply the $k$-th, $k \in\left[1, N_{r}\right], \mathbf{w}_{r}$ to $N_{d}$ OFDM symbols with indexes $k, k+N_{d}, \cdots, k+\left(N_{r}-1\right) N_{d}$. This interleaved variation of $\mathbf{w}_{r}$ is mainly to enable better estimation of Doppler shift, which is very small and needs to be measured over a long period. The set of $N_{r}$ different $\mathbf{w}_{r} \mathbf{s}$ can be either random vectors fulfilling compressive sensing requirement or vectors generating directional beams pointing to $N_{r}$ desired scanning directions; and

(3) For communications, $\mathbf{w}_{r}$ for the two arrays shall be ideally jointly designed to achieve the optimal results. Due to their orientation difference, this could be very challenging. A suboptimal approach is to design the beamforming for each array independently and then apply a single phase shift to the sum signal for one array to ensure a constructive combining of signal energy from two arrays. If no passive sensing is required, each array can simply generate a single beam pointing to the transmitting node; otherwise, $\mathbf{w}_{r}$ needs to be fixed during one packet to keep channel fixed, and hence no scanning is implemented (Detailed design and implementation will not be discussed in this paper).

Therefore one complete scanning requires $N_{t}$ packets and $N_{t} N_{r} N_{d}$ OFDM symbols. The width and gain for the sensing and communication beams can be designed to adapt to these parameters. Optimization of $N_{t}, N_{r}$ and $N_{d}$ is yet to be investigated.

Note that the above interleaved design of $\mathbf{w}_{r}$ is specific to the requirement of estimating the Doppler shift using the compressive sensing technique. It can be varied when needed.

\section{Receiver Signal Processing - Sensing Stage}

The basic task in sensing is to determine distance, direction, and speed of environmental objects from received signals, and then extract information from these parameters. They can be obtained through both direct estimation of parameters $\tau_{\ell}, f_{D, \ell}$, $\theta_{t, \ell}$ and $\theta_{r, \ell}$ and data fusion processing over time, frequency and spatial domains. In this paper, we only provide basic formulations for estimating these parameters without going to detailed design and optimizations.

Since $\tilde{\mathbf{s}}$ is known to the receiver and $\mathbf{w}_{t}$ and $\mathbf{w}_{r}$ are fixed for at least one OFDM symbol, we can convert the received signal 
to the frequency domain and remove $\tilde{\mathbf{s}}$ via equalization. Ignore the variation of Doppler phase shift over $\left[k T_{s},(k+1) T_{s}\right]$ and

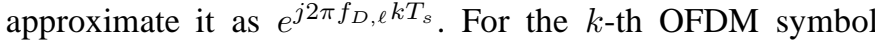
$\left(t=k T_{s}\right)$ we can get the frequency-domain channel estimate at the $n$-th subcarrier as

$$
\begin{gathered}
\tilde{h}_{n, k}=\sum_{\ell=1}^{L} \underbrace{\left(b_{\ell}\left(\mathbf{w}_{t}^{T}(k) \mathbf{a}\left(\theta_{t, \ell}\right)\right) \mathbf{w}_{r}^{T}(k) \mathbf{a}\left(\theta_{r, \ell}\right)\right)}_{g_{\ell, k}} . \\
e^{-j 2 \pi n \tau_{\ell} f_{0}} e^{j 2 \pi k f_{D, \ell} T_{s}}+\tilde{z}_{n} / \tilde{s}_{n},
\end{gathered}
$$

where $\tilde{z}_{n}$ is the noise sample at the $n$-th subcarrier.

For fixed $\mathbf{w}_{t}$ and $\mathbf{w}_{r}, g_{\ell, k}$ will be a function of $\ell$ and the signal in (5) presents a form of typical radar signal processing. The parameters $\tau_{\ell}$ and $f_{D, \ell}$ can be coarsely estimated by using simple 2D DFTs, across frequency (subcarriers) and time (OFDM symbols), respectively [5]. However, this approach generally achieves very limited resolution.

Resolution for $\tau_{\ell}$ and $\mathbf{f}_{D, \ell}$ can traditionally be improved using super-resolution spectrum analysis techniques such as periodogram and ESPRIT [5]. More recently, compressive sensing (CS) techniques have been widely applied in radar imaging [6], [7]. The four parameters, AoDs, AoAs, delay and Doppler frequency in (5) can be solved either individually or jointly by forming from 1D to 4D CS models. 2D CS formulation is the typical model used for radar [6], [7]. High-order CS formulation using the Tensor tools is also emerging. However, our problems here involve overcomplete dictionaries for estimating all these parameters due to their continuous nature and small spanning in respective domains. Since higher dimension CS algorithms for solving problems with overcomplete dictionaries are not mature yet, we propose a novel 1D Bayesian Compressive Sensing (BCS) formulation here which is powerful for solving such problems and could promise lower complexity than its higher order peers.

For a BCS formulation, there can only be one common weight applied to the column in the dictionary matrix. This limits the ways of formulating the BCS problems, and is the main reason for the interleaved design of $\mathbf{w}_{r}$ in Section III-B.

To estimate $\tau_{\ell}$, we can treat the Doppler phase term and $g_{\ell, k}$ in (5) as varying amplitude over different measurements for the delay phase term, and then formulate it as a multiple measurement vector (MMV) BCS problem. This MMV forumulation has an overcomplete dictionary of interpolated DFT matrix with dimension $N \times p_{r} N$ where $p_{r}$ is the interpolation factor. The observation matrix can be as large as $N \times\left(N_{r} N_{d}\right)$, with signals from $N$ subcarriers across $N_{r} N_{d}$ consecutive OFDM symbols. Every $N_{r} N_{d}$ symbols will generate one MMV estimate for $\tau_{\ell}$ and the corresponding amplitudes.

The estimation of $f_{D, \ell}$, AoAs and AoDs can be similarly formulated across different domains of signals. Due to the limited number of rows in the observation matrices, our preliminary simulation results show that their estimates are not as accurate as the delay estimates for which the number of rows is a large $N$. Hence, we propose a different novel approach that directly estimates $f_{D, \ell}$ and AoAs from the estimated amplitudes in the MMV outputs for $\tau_{\ell}$.
Let $\mathbf{Q}$ be the output matrix of size $N \times\left(N_{r} N_{d}\right)$ in the delay MMV formulation. In noise-free cases, its elements in the $L$ non-zero rows shall be equal to

$$
q_{\tilde{\ell}, k}=g_{\ell, k} e^{j 2 \pi k f_{D, \ell} T_{s}}, k=1, \cdots, N_{r} N_{d},
$$

where $\tilde{\ell}$ is related to the dictionary size and does not necessary equal to $\ell$. Note that $g_{\ell, k}$ is a periodic function of $k$ of period $N_{r}$ according to our choice of $\mathbf{w}_{r}$. Hence for $q_{\tilde{\ell}, k}, q_{\tilde{\ell}, k+N_{r}}, q_{\tilde{\ell}, k+2 N_{r}}, \cdots$, we can compute their phase differences to get an estimate of $f_{D, \ell}$. A threshold for the averaged cross correlation of $q_{\tilde{\ell}, k}$ can be used to extract efficient estimates.

Different amplitude estimates can be obtained from different $\mathbf{w}_{r}$ s scanning the directions close to the AoA of each object. A grid graph of power-distance-direction that maps the power of averaged cross correlation of $q_{\tilde{\ell}, k}$ to $2 \mathrm{D}$ distance-direction grids can be obtained and used for determining AoA in relation to distance. Furthermore, the maximal amplitude estimate in general will correspond to the actual direction if no two objects are at the same distance. Hence we can apply a simple approach Peak-Picking to filter the results, which picks up the one with the maximal power as the AoA estimate from all the $\mathbf{w}_{r}$ scanning directions for each delay estimate.

There could be potentially various implementations and improvements based on the MMV formulations above, such as parameter optimization, complexity reduction, interpretation of power-distance-direction graph and off-grid adaptation. These are our ongoing research topics.

\section{Simulation Results}

We present some preliminary simulation results to validate the proposed framework. We consider a system with parameters detailed in Section III-A where $N_{t}=N_{r}=N_{d}=8$, $L=8$. The transmission power is $40 \mathrm{dBm}$. No radar crosssection information is assumed and this example is purely based on free-space pathloss. We assume a free-space pathloss model with path-loss factor 4 for sensing, and the total thermal noise in the receiver is $-95 \mathrm{dBm}$. The vehicle is moving at speed $40 \mathrm{~m} / \mathrm{s}$, and obstacles have random speed between 20 and $20 \mathrm{~m} / \mathrm{s}$. The interpolated DFT matrix is used as the dictionary with an interpolation factor of 4 . Delays are on grid and correspond to a distance resolution of $0.93 \mathrm{~m}$. Continuous values are used for speed and hence Doppler shift. Both AoAs and AoDs are on grid with a resolution of 2.8 degrees. We use 64 different $\mathbf{w}_{r} \mathrm{~s}$ (phase-only) to generate directional beams to scan 64 directions spaced at 2.8 degrees, over 8 packets.

We use the iterative two-step least squares method in [8] to generate two beams simulatanously, with one beam fixed to a direction and the other one scanning a range of about 180 degrees. In Fig. 3 we show such beams for a 8-antenna array. To generate such beams, $\mathbf{w}_{t}$ uses both continuous magnitude and phase values.

We present estimation results for $\tau_{\ell}, f_{D, \ell}$ and AoAs from a random implementation using 512 OFDM symbols here. Fig. 4 demonstrate the power-distance-direction plot. Figs. 5 and 6 show the estimation results for distance-AoA, and relative speed-distance, respectively. In all figures, red circles are for 


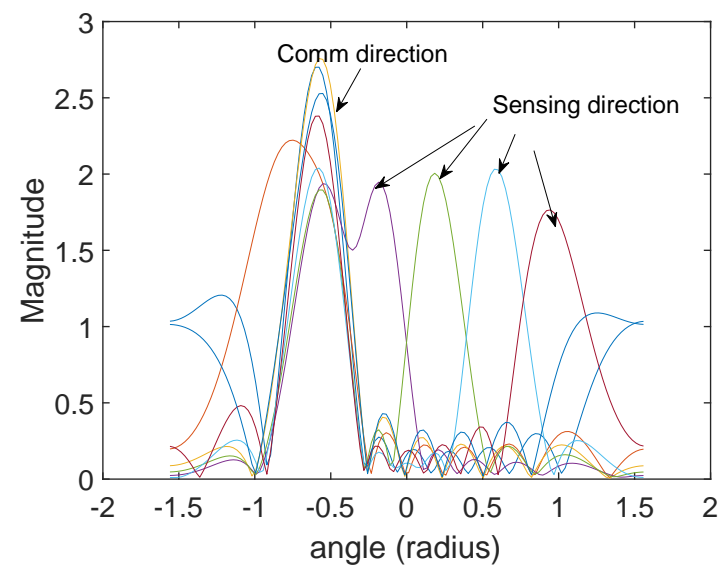

Fig. 3. Multibeam for communications and sensing. One beam is fixed to t communication direction. The other beam scans multiple sensing direction

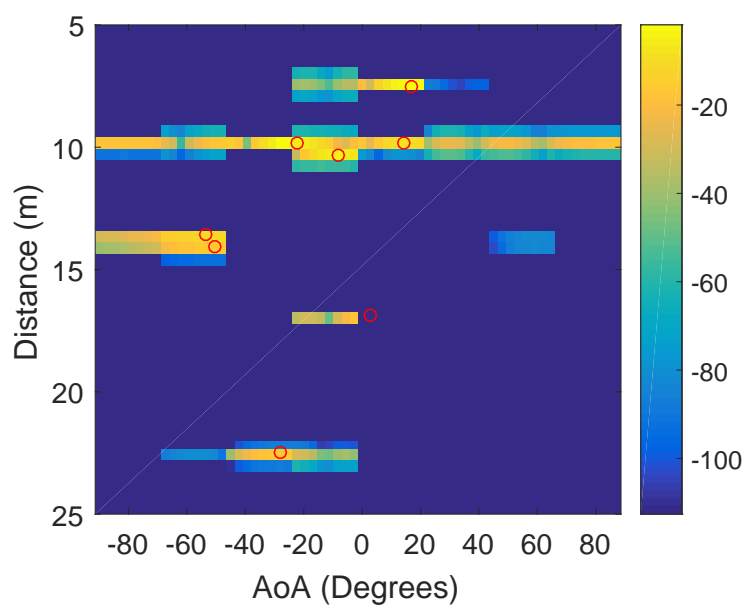

Fig. 4. Power-distance-direction plot where colormap shows power in $\mathrm{dB}$.

actual values, and blue crossings are for the estimates. Fig. 5.(a) presents the original distance estimates for $N_{t}$ scanning directions of $\mathbf{w}_{t}$. Fig. 5.(b) demonstrates the cleaned results after applying the Peak-Picking approach. In this particular example, there is one mis-excluded object because two objects locate at the same resolved distance bin. Fig. 6 demonstrates the corresponding speed estimation.

\section{CONCLUSIONS}

We have presented a framework of using beamforming with two steerable analog antenna arrays for joint communications and sensing. We demonstrated that using the proposed technique, it is possible to integrate sensing into OFDM communications using two directional beams for transmission, and a single scanning beam for receiver sensing, without requiring special packet structures. Simulation results validate that the proposed novel 1D Bayesian compressive sensing can efficiently resolve closely spaced objects and their moving speed within the framework. Among many possible improvements, better post-processing to extract valid AoAs from the power-distance-direction graph is a priority research problem. (a)

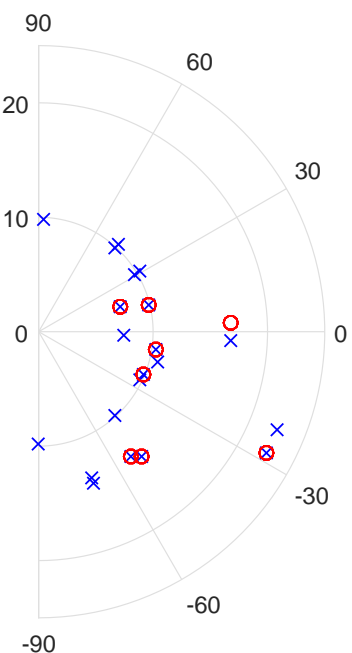

(b)

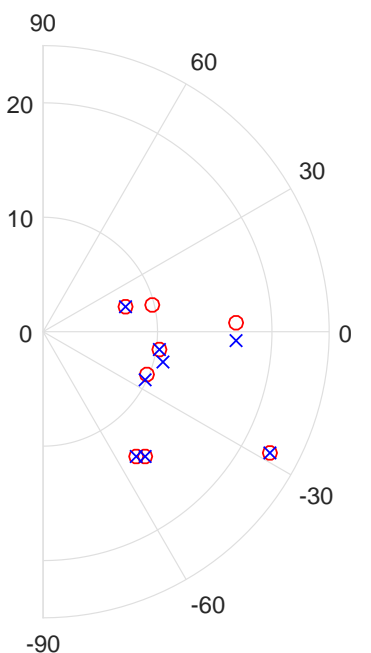

Fig. 5. Estimation of the distance (meter) and AoAs (degrees).

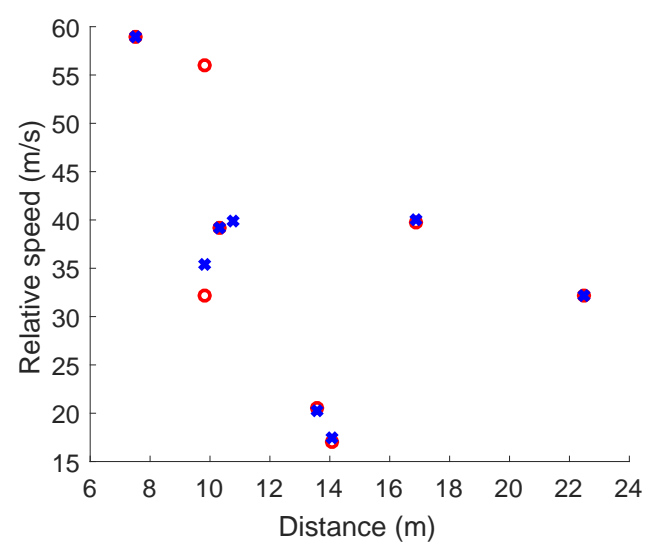

Fig. 6. Estimation of the distance (meter) and speed $(\mathrm{m} / \mathrm{s})$.

\section{REFERENCES}

[1] L. Han and K. Wu, "Joint wireless communication and Radar sensing systems - state of the art and future prospects," IET Microwaves, Antennas Propagation, vol. 7, no. 11, pp. 876-885, August 2013.

[2] P. Kumari, N. Gonzalez-Prelcic, and R. W. Heath, "Investigating the IEEE 802.11ad standard for millimeter wave automotive radar," in VTC Fall, 2015 IEEE 82nd, Sept 2015, pp. 1-5.

[3] N. González-Prelcic, R. Méndez-Rial, and R. Heath Jr., "Radar aided mmWave beam alignment in V2I communications supporting antenna diversity," in Information Theory and Applications Workshop (ITA), January 2016.

[4] C. Sturm and W. Wiesbeck, "Waveform design and signal processing aspects for fusion of wireless communications and radar sensing," Proceedings of the IEEE, vol. 99, no. 7, pp. 1236-1259, July 2011.

[5] K. M. Braun, "Thesis: OFDM radar algorithms in mobile communication networks," 2014. [Online]. Available: http://digbib.ubka.unikarlsruhe.de/volltexte/documents/2987095

[6] M. A. Herman and T. Strohmer, "High-resolution radar via compressed sensing," IEEE Transactions on Signal Processing, vol. 57, no. 6, pp. 2275-2284, June 2009

[7] M. A. Hadi, S. Alshebeili, K. Jamil, and F. E. A. El-Samie, "Compressive sensing applied to radar systems: an overview," Signal, Image and Video Processing, vol. 9, no. 1, pp. 25-39, 2015.

[8] Z. Shi and Z. Feng, "A new array pattern synthesis algorithm using the two-step least-squares method," IEEE Signal Processing Letters, vol. 12, no. 3, pp. 250-253, March 2005. 